Colomo Magaña, E. \& Cívico Ariza, A. (2018). La necesidad de formación del profesorado en pedagogía de la muerte. Revista Electrónica Interuniversitaria de Formación del Profesorado, 21(1), 83-94.

DOI: http://dx.doi.org/10.6018/reifop.21.1.279961

\title{
La necesidad de formación del profesorado en pedagogía de la muerte
}

\author{
Ernesto Colomo Magaña, Andrea Cívico Ariza \\ Universidad Internacional de Valencia (VIU)
}

\section{Resumen}

Aunque la muerte forma parte de la vida, aún no se ha conseguido abordar plenamente desde la perspectiva pedagógica. Debido a ello, este trabajo se centra en la percepción que tienen los estudiantes del Máster de Secundaria en relación a su nivel y necesidad de formación sobre el tema de la muerte. Se ha llevado a cabo mediante la elaboración, análisis e interpretación de autoinformes sobre la experiencia y conocimientos de la muestra participante. Los resultados evidencian que los alumnos de la Universidad Internacional de Valencia han recibido una escasa formación pedagógica respecto a la muerte, aunque la consideran fundamental. Tras los resultados, se plantea la utilización de los dilemas y el role playing como una propuesta metodológica desde la que es posible desarrollar esta temática. Las conclusiones incluyen una reflexión sobre la importancia de abordar la Pedagogía de la Muerte como una necesidad para la formación del profesorado.

Palabras clave

Pedagogía de la muerte; formación de profesores; currículo; material didáctico.

\section{The need for training teachers in death pedagogy}

\begin{abstract}
Although death is a part of live, it has not yet been able to fully address it from the pedagogical perspective. Because of that, this paper focuses on the perception of Master's of Secondary Education students in relation to their level and need for training on the subject of death. It has been carried out through the development, analysis and interpretation of self-reports about the experience and knowledge of the participating
\end{abstract}

Contacto:

Ernesto Colomo Magaña, ernesto.colomo@campusviu.es, Universidad Internacional de Valencia (VIU), España. 
sample. The results show that the students of the International University of Valencia have received little pedagogical training regarding death, although they consider it fundamental. After the results, the use of dilemmas and role playing as a methodological proposal from which it is possible to develop this theme. The conclusions including a reflection about the importance of addressing the Pedagogy of Death as a necessity for teacher training.

\section{Key words}

Death education; teacher training; curriculum; teaching aid.

\section{Introducción}

Pese a los avances logrados a nivel educativo y pedagógico, el espacio para muchas de las preguntas que se hace la humanidad (muerte, conciencia, espiritualidad, etc.) sigue sin tener un planteamiento definido. Entre dichos ámbitos, la finitud es uno de los contenidos que presenta cierta resistencia para ser abordado pedagógicamente, pese a que las personas son los únicos seres vivos capaces de reflexionar sobre su mortalidad (Rodríguez, Herrán y Cortina, 2015b). Igual que se pregunta de dónde viene, quiere saber a dónde va, pero la angustia por la incertidumbre y el desconocimiento hace que postergue, e, incluso, intente obviar dicha situación, pues la muerte inquieta al hombre en todos los sentidos. En la actualidad, existe dificultad para hablar sobre esta realidad porque se ha desnaturalizado. Antaño, el vivir diferentes experiencias en torno a este fenómeno solventaba muchas de las cuestiones que hoy el desconocimiento genera: ¿cómo se explica qué es la muerte?, ¿cómo se cuenta que no se volverá a ver, tocar, oír ni sentir a la persona fallecida?, ¿qué discurso o palabras se eligen para acompañar y no dañar? En definitiva, falta formación y herramientas para hablar de la muerte a nivel educativo. Cortina (2010, p. 58), sostiene que "si la educación es formación, introducir la muerte en los estudios es proveerles de una perspectiva más cierta e intensa de la vida, es dotarla de recursos existenciales". Partiendo de esta afirmación, existen estudios internacionales (Engarhos, Talwar, Schleifer y Renaud, 2013) centrados en la reflexión sobre la necesidad de formación para los educadores en el ámbito de la muerte. Sin embargo, la educación oficial en España hace como si la muerte no existiera y la relega del ámbito formativo.

No podemos tampoco olvidar que, desde niños, hemos sido educados y entrenados en multitud de asuntos, algunos de ellos un tanto irrelevantes, pero el tema de la muerte parece invisible, tanto por parte de los padres como en el colegio e, incluso, en la educación superior. (Gaona, 2015, p. 23).

En lugar de reconocer a la muerte como el episodio final y la bajada del telón (Sampedro, 2015), se educa al margen de ese momento y de la condición mortal. Esta situación hace que los profesionales de la educación tengan escasa o nula formación sobre cómo tratar el final de la vida a nivel pedagógico. Por este motivo, es tan importante poder normalizar la muerte como fenómeno humano y potencialmente educativo. Teniendo en cuenta estas premisas, en este artículo el objetivo principal es valorar la necesidad de formación respecto a la Pedagogía de la Muerte en los profesionales de la educación. Entre los objetivos específicos se contempla: a) destacar la importancia de la formación y la innovación pedagógica en el ejercicio de la profesión docente; b) conocer las diferentes herramientas e instrumentos pedagógicos para trabajar la muerte a nivel educativo; c) plantear los dilemas y el juego de roles como una metodología didáctica apta para la formación del profesorado en Pedagogía de la Muerte. 


\section{Formación del profesorado y Pedagogía de la Muerte}

La enseñanza y la formación son el pilar del conocimiento, siendo el desafío actual de la educación el preparar a las futuras generaciones para su adaptación a la complejidad de la vida personal, social y profesional. Este hecho conlleva, necesariamente, una revolución en la formación de los propios educadores, convirtiéndose en catalizadores de los procesos de enseñanza-aprendizaje (Pérez Gómez, 2010). Tanto la figura del profesor como la calidad, desarrollo y perfeccionamiento del mismo es determinante en la educación del alumno. Esta situación hace que se considere necesaria, como una exigencia de la propia naturaleza de la profesión docente, la formación continua. La vida está en constante transformación, fruto de la globalización y de los avances en las distintas ramas del conocimiento, lo que obliga a estar actualizados. Además, el ser humano se caracteriza por su factor evolutivo, siempre abierto a seguir perfeccionándose y mejorando, por lo que nunca se llega a estar completos desde el punto de vista del aprendizaje, es decir, que "nadie está formado para siempre" (Santos Guerra, 2010, p. 175). Pese a esta necesidad, ni la universidad española ni las facultades de Ciencias de la Educación se encuentran cercanas al ideal que supone la formación de profesionales docentes competentes para la tarea que reclama la educación del siglo XXI. Siguen caracterizándose por ser conservadoras y tradicionales, promoviendo una formación dirigida a la mejora de los aprendizajes del alumnado a tenor de resultados cuantificables (Lupión y Gallego, 2017), siguiendo cerradas a la innovación en determinas áreas. Sin embargo, la adaptación a la realidad actual conlleva una apertura a nuevos contenidos, metodologías, vínculos y sinergias educativas a través de temas innovadores que favorezcan la evolución humana. Todo proceso de enseñanza-aprendizaje del docente centrado solo en lo cognitivo y en los resultados académicos proporcionará una formación fragmentada y parcelada de la realidad. Si se quiere un desarrollo integral, global y holístico del alumnado, se deben incluir todos aquellos elementos/ámbitos que favorezcan dicho proceso, de lo contrario se obtendrá una formación reduccionista.

Esto es lo que ha acontecido en torno a la cuestión de la muerte y la educación, dando lugar a una situación de rechazo entre dos elementos cuya relación se antoja clave para el desarrollo identitario de los discentes. Ante dicho escenario, se considera que este tema debe ser uno de los ámbitos que formen parte de la innovación curricular en la formación de los educadores, ya que la muerte suscita interés, curiosidad e incertidumbre en el alumnado (Bowie, 2000). Por lo tanto, no se puede dejar que un fenómeno que afecta a la vida, llegando a determinarla, siga siendo ignorado a nivel educativo y no forme parte ni de los procesos de enseñanza-aprendizaje del alumnado ni de la formación inicial del profesorado (Veneu y Costa, 2016). Debe tener un espacio propio, apareciendo aquí como propuesta la pedagogía de la muerte. Esta representa el área, dentro del ámbito educativo, que ha sido capaz de profundizar en esta temática, aunque está carente de tradición profesional en didáctica y pedagogía (Rodríguez, Herrán y Cortina, 2015b). Tiene como ejes dos evidencias comprobadas que acompañan al momento de la muerte. Por un lado, la finitud del ser humano, al vivir en ciclos constantes de nacimientos y muertes que permiten una evolución y transformación como especie. Por otro lado, la circunstancia de que la muerte sea un hecho inevitable, personal e intransferible cuya certeza reside en el interior de cada sujeto. Asimilar la mortalidad es un proceso de enseñanza-aprendizaje continuo que se desarrollará durante toda la vida, cuyos resultados irán vinculados a la madurez personal de cada uno. No obstante, alejarse de visiones trágicas y del miedo a perder la vida naturalizará este momento y permitirá disfrutar más del tiempo y del sentido que cada uno de a su existencia. Entre los contenidos de la pedagogía de la muerte no solo se aborda la normalización del final de la vida, sino que también se incluyen elementos que permiten aprender a vivir sin obviar dicha realidad, sirviéndoles a las personas para comprender su 
papel, su condición, su igualdad con el resto y su fin. Igual que se aprende a vivir, se debe aprender a morir, a estar preparado para un viaje que se inicia en el nacimiento y que cuyo fin inevitable, permita a las personas orientar positivamente sus vidas.

\section{Metodología}

Esta investigación analiza la percepción sobre el nivel y necesidad de formación sobre el tema de la muerte y los diferentes recursos y estrategias existentes para trabajarlo a partir de los autoinformes elaborados por alumnos de la Universidad Internacional de Valencia (VIU).

\section{Muestra}

La muestra objeto de estudio estará conformada por estudiantes matriculados durante el curso académico 2016/2017 (edición de abril) en el Máster Universitario en Formación del Profesorado en Educación Secundaria Obligatoria, Bachillerato, Formación Profesional y Enseñanza de Idiomas, en las asignaturas de «Educación Emocional y Habilidades Sociales. La empatía»; «Procesos y Contextos Educativos»; y «Sociedad, Familia y Educación». Partiendo de que la población a la que se dirigía este estudio estaba compuesta por los estudiantes matriculados en el Máster de Secundaria de la VIU (1100 plazas edición de abril), la muestra ha quedado finalmente conformada por 250 estudiantes de esta titulación $(n=250)$, representando a un $22,7 \%$ de la población de referencia. Se trata de una muestra seleccionada de manera intencionada (no probabilística) directamente condicionada por el ejercicio de la docencia en estas materias por parte de los investigadores. Este grupo no pretende ser representativo a nivel estadístico, ya que su función es exploratoria, expresiva e indicativa de las principales consignas recogidas en los autoinformes sobre el ámbito de la Pedagogía de la Muerte.

\section{Instrumento}

Respecto al autoinforme, se trata de un documento informativo proporcionado por el propio individuo (producido de manera voluntaria por petición del tutor de las asignaturas) a partir de unas preguntas alojadas en el foro de la materia en la que estuvieran matriculados. Las ventajas que presenta esta herramienta para el estudio, radica en que las opiniones son recogidas a partir de lo que expresan por escrito los sujetos de un modo impersonal, ahorrando mayor tiempo que con otros instrumentos como la entrevista. En este caso, los participantes confeccionan sus respuestas sobre el nivel y necesidad de formación sobre la Pedagogía de la Muerte y qué herramientas/recursos/materiales pueden utilizarse para abordar esta temática en el aula, siendo estas las preguntas (de respuesta abierta) que se proponían. Al ser las respuestas subidas a los foros y haberse distribuido digitalmente, se trabaja con un instrumento poco costoso y fácil de aplicar simultáneamente, permitiendo su extensión a una muestra amplia.

\section{Procedimiento}

Tras la recopilación de todos los autoinformes, se realiza un análisis y estudio de los mismos desde la perspectiva nomotética. La intención es poder percibir y entender las opiniones aportadas por los sujetos de la muestra, categorizando todos los pensamientos y perspectivas expuestas (tanto de manera cuantitativa como cualitativa). Para ello, se parte del análisis de contenido como instrumento de trabajo, donde se combina la observación (lectura) y la producción de datos (interpretaciones), desarrollando las siguientes fases (Piñuel y Gaitán, 1995): pre análisis, donde se organiza el material informativo; creación del sistema categorial, donde se construye la herramienta con la que se ordenará la información 
para su análisis posterior; codificación, donde se transforman los contenidos en unidades más sencillas para describirlos posteriormente; interpretación (será la fase que se recoja en la investigación), donde se otorga significado al material informativo obtenido de los autoinformes. En las interpretaciones sobre el material aportado por los sujetos de la muestra, se utiliza el construccionismo. Se parte de la idea de que no hay una única visión objetiva sobre cómo educar respecto a la muerte, si no que esta se construye y depende de múltiples factores (nivel evolutivo, ritmo de aprendizaje, contexto, realidad, etc.). Por esta razón, se trabaja siempre abiertos al debate, la reflexión y la reconstrucción de los mismos.

\section{Resultados}

En este apartado se pone de manifiesto la opinión y propuestas de los futuros profesionales de la educación en torno a la inclusión de la pedagogía de la muerte como contenido para su formación. Se comienza abordando en el análisis de los autoinformes la formación recibida sobre este ámbito y su consideración sobre la relevancia de la misma; posteriormente, se expone una enumeración de algunos estudios sobre herramientas e instrumentos para trabajar la muerte en las aulas; y se finaliza con una propuesta didáctica con carácter formativo para trabajar esta temática.

No podemos enseñar aquello que desconocemos.

El análisis de los datos obtenidos de los autoinformes en torno a la formación recibida sobre el tratamiento pedagógico de la muerte y el duelo es el punto de partida.

Tabla 1.

Formación pedagógica recibida respecto a la muerte $(n=250)$.

\begin{tabular}{|l|l|}
\hline Formación pedagógica respecto a la muerte & $\%$ \\
\hline Formación recibida de carácter religioso & 8,0 \\
\hline Formación recibida en el ámbito universitario & 4,0 \\
\hline Ninguna formación recibida & 88,0 \\
\hline
\end{tabular}

La muerte, pese a ser un suceso natural, sigue siendo un tema tabú, constantemente vetado en el ámbito educativo y apartado de nuestra cotidianeidad (Herrán y Cortina, 2007), no siendo abordado como contenido en la formación inicial de los profesionales de la educación.

"No debemos educar con tabúes y ocultando fases naturales de la vida, ya que a la larga produce mayor dolor y sufrimiento no hablar de estos temas" (S. 111).

La escasa formación que han recibido los participantes de la investigación (tabla 1) proviene de su formación inicial en algunas áreas de estudio no vinculadas directamente con el mundo educativo (todos los casos de la muestra habían cursado el grado/licenciatura en Psicología). El resto habían recibido formación dentro del ámbito religioso (parroquia, catequesis, estudios teológicos), asegurándoles una vida después de la muerte (algo no sustentado científicamente de momento) y una justicia divina, que separa a los justos que van al cielo de los injustos que van al infierno por haber tenido una vida moralmente cuestionable (Gaona, 2012a). Las ideas religiosas en torno al fallecimiento son bastante 
difundidas y aceptadas, provocando así una visión sesgada y no neutra sobre el final de nuestra existencia.

"He recibido formación religiosa en el ámbito de la muerte, que no encuentro relevante excepto en educación religiosa, ya que, en realidad, no explica nada y es un consuelo meramente. No se nos educó en la gestión de las emociones: por qué nos sentíamos tristes, cómo debíamos gestionarlo, cómo ha afectado esta muerte a nuestra relación con nuestra familia y con los demás, etc., con lo que no resultaba realmente efectivo. Creo que una formación en este sentido hubiera sido realmente útil, y nos hubiera enseñado a gestionar nuestras emociones y sentimientos de forma más sana y equilibrada que simplemente "confiando en Dios". (S. 221).

Como evidencia este argumento, desde el ámbito educativo se debe favorecer una enseñanza global y holística sobre este fenómeno, aportando respuestas, herramientas e instrumentos para poder afrontar la finitud de la vida desde una comprensión emocional y cognitiva positiva.

Tabla 2.

Valoración sobre la necesidad de formación pedagógica respecto a la muerte $(n=250)$.

\begin{tabular}{|l|l|}
\hline Percepción sobre la necesidad de formación & $\%$ \\
\hline No necesaria & 0,0 \\
\hline Si necesaria & 100,0 \\
\hline
\end{tabular}

Dentro de la misma cuestión, se pregunta a los participantes si consideran necesaria dicha formación como futuros profesionales de la educación (tabla 2). Los resultados muestran unanimidad (100\%) en que se trata de una formación necesaria para desarrollar su labor docente.

No se puede obviar que educar es dotar de herramientas e instrumentos al alumnado para que puedan enfrentarse y resolver los diferentes problemas que se les presenten en su vida y su realidad. En este sentido, la muerte tiene un potencial formativo inherente para el desarrollo de competencias emocionales que permitan gestionar estas situaciones y para el consiguiente desarrollo personal (Márquez-Cervantes, y Gaeta-González, 2017). Por esta razón, entre las misiones del docente está el acompañamiento al educando durante su proceso de (re)construcción identitaria, siendo piezas claves para su desarrollo integral, evolución y crecimiento.

"Como futuros docentes nuestra labor traspasará las propias fronteras de la materia que impartamos y este es uno de los aspectos sobre los cuales debemos tener herramientas para ofrecerlas a los alumnos con la finalidad de que, al margen de trabajar la parte más intelectual de su persona humana, sepan afrontar situaciones que inefablemente ocurrirán en sus vidas" (S.137).

Es fundamental que se tengan los conocimientos necesarios para no cometer errores que puedan dificultar el proceso. La formación de los educadores en pedagogía de la muerte permitirá abordarla en cualquier contexto educativo, enseñando que la muerte es parte de la vida (trabajo preventivo) y acompañando al alumnado en sus procesos de duelo (trabajo paliativo). Como educadores no se puede enseñar aquello que se desconoce, por lo que se 
debe estar formado y preparado para dar respuestas al interrogante del tema de la mortalidad.

"¿Cómo puedo ayudar a un alumno a superar un proceso de muerte de un ser querido si no tengo suficiente formación?" (S. 138).

Para poder enseñar algo hay que conocerlo en profundidad, debiendo asimilar bien los conocimientos para su interiorización y construcción de manera personal, teniendo la obligación de subsanar y cubrir dichas lagunas formativas.

"Para que la pedagogía de la muerte se implemente en los centros de enseñanza, es necesario que los educadores sean formados en la misma, ya que muchos desconocen las pautas a seguir para abordar este tema tan delicado que conecta con los miedos más elementales del ser humano" (S.215).

"Es conveniente poseer las herramientas y habilidades necesarias que nos posibiliten ayudar a asumir situaciones dolorosas e irreversibles, lo más urgente y básico es tener la capacidad de acompañar, escuchar, tranquilizar y apoyar a los que sufren" (S.120).

El fin de que las personas comprendan su mortalidad y acepten las pérdidas que sufrirán está dirigido a que reflexionen sobre la importancia de la vida.

"Veo esencial formar al individuo en la aceptación de la muerte como fase final de la vida. No se puede vivir atemorizado por el miedo a la muerte, ya que de esta manera no apreciaremos la vida en su plenitud" (S. 13).

Lo importante y que se debe trasladar como educadores no es la incertidumbre de si hay vida después de la muerte. Lo que es seguro es que hay vida antes de la muerte, por lo que al perder el miedo a la muerte se da más valor a la vida y a los actos que se hagan durante la misma. Así, se incrementa tanto el placer de vivir con mayor plenitud cada momento (Gaona, 2012b, 2015), como el compromiso y la responsabilidad hacia las decisiones y actos (Herrán y Cortina, 2007).

Herramientas e instrumentos para acercar la muerte a la educación: los dilemas y juegos de roles.

Otro aspecto trabajado a través del autoinforme fue la elaboración de un listado con herramientas e instrumentos que se pudieran utilizar para abordar la pedagogía de la muerte a nivel educativo. Partiendo de considerar esta formación como fundamental, el tener diferentes recursos que permitan aplicar estos conocimientos para su desarrollo en el aula también se convertía en un factor clave.

"El uso de material educativo que trate la muerte les puede ayudar a entender el tema como un proceso natural" (S. 111).

Rodríguez, Herrán y Cortina (2015a) ofrecen un listado de técnicas de enseñanza y propuestas educativas para la pedagogía de la muerte, desde una perspectiva holística y transdisciplinar, con las que se podrían abordar los diferentes contenidos y situaciones adaptándose al nivel educativo y las características cognitivas y socioemocionales del alumnado. Entre las propuestas más frecuentes (a tenor de los estudios realizados en los últimos años) y coincidentes con las extraídas de los autoinformes de la muestra, destacan el uso del cine (Cortina, 2010; Niemiec y Schulenberg, 2011); la literatura (Colomo, 2016; Selfa, Fraga y Berengué, 2015); la música (Colomo y Oña, 2014); el aprendizaje servicio (Rodríguez, Herrán y Cortina, 2015b); o las representaciones artísticas (Rodríguez y 
Gorrayola, 2012). Estas experiencias que se trasladan sobre la muerte repercuten en las personas que las reciben, aprendiendo conocimientos y premisas de modo indirecto a través de la imitación/rechazo de las conductas expuestas (Bandura, 1982). Se convierten así en recursos que influyen en las formas de pensar, sentir y actuar en torno a la realidad de la mortalidad.

No obstante, se quiere profundizar en un instrumento que, pese a su habitualidad, no ha tenido el mismo desarrollo que otras herramientas a nivel de investigaciones y propuestas. Se trata de plantear dilemas y situaciones de juegos de roles (role playing), que permitan trabajar con grupos de discusión y de debate. En ellos la reflexión, el análisis y el desarrollo del pensamiento crítico jugarán un papel fundamental. Se trata de una técnica cuyo potencial reside en el valor educativo que el diálogo y el razonamiento pueden aportar tanto para la comprensión de la finitud de la vida (prevención) como para el acompañamiento tras una pérdida (paliativo).

"Hablar sobre lo que nos preocupa, sobre lo que nos depara la vida, es importante para intercambiar opiniones y experiencias que nos permitan dar un paso al frente" (S. 19).

Para ello, se parte de los dilemas como herramienta de trabajo. Se trata de pequeñas historias en las que en un contexto real, se plantea una situación posible pero conflictiva a nivel moral (Grup Xibeca, 1995). El uso de dilemas proporciona al alumnado tiempo para reflexionar, problemas que despiertan su interés y otros compañeros con los que debatir y pensar (Daniels y Bizar, 2005). Se trata de construir escenarios ficticios donde el educando tiene que elegir qué solución es la más acertada o bien analizar los pros y los contras de la decisión que haya elegido el protagonista. De esta manera, se sitúa a los individuos ante situaciones conflictivas, que les hacen replantearse y transformar aquellas normas, protocolos, actitudes, valores y comportamientos morales que ellos siguen para ver si son las más acertados o puede haber decisiones mejores que las que ellos suelen utilizar, promoviendo así una relación entre experiencia y saber (Contreras, 2010). Se trabaja así el control y gestión emocional de los discentes ante el tema de la muerte, favoreciendo una comunicación asertiva entre ellos que les dote de recursos y estrategias para reducir los miedos y la ansiedad ante la condición mortal. Se podrán utilizar dilemas creados para abordar estas temáticas o de redacción propia, lo que supone un reto a través del cual el docente puede desarrollar su competencia de aprender a aprender (el buen maestro nunca deja de ser alumno) y le permita crear un material que se adapte a la realidad de su aula.

Para crear el dilema, se debe abordar la muerte de forma que sea asumible emocional y cognitivamente, teniendo en cuenta los ritmos, preocupaciones, necesidades, nivel de desarrollo y capacidades del alumnado para dotar de significado a esta realidad. Partiendo de los conocimientos previos de los educandos, como base para el proceso de enseñanzaaprendizaje, se introducirán conceptos claves sobre el hecho de perecer desde una perspectiva holística, de manera que se produzca un desequilibrio cognitivo entre lo que saben y la nueva información. A partir de la reconstrucción de sus esquemas conceptuales, donde incluirán lo que se les haya enseñado, se favorecerá el reequilibrio entre ambas realidades, de manera que los discentes aprendan de manera significativa todo lo concerniente a recursos y estrategias socioemocionales en torno a la muerte. La clave es que puedan transferir dichos conocimientos a diferentes contextos en los que precisen de los mismos.

A continuación, se plantea el diseño de un dilema para debatir mediante un role playing dentro de la etapa universitaria durante la formación inicial/continua de los profesionales de la educación. 


\section{Propuesta didáctica de pedagogía de la muerte desde los dilemas y el juego de roles.}

Se pretende profundizar y reflexionar sobre el papel que los diferentes agentes educativos pueden jugar ante una situación compleja como es una muerte. La tarea propuesta va a ser la realización de un role playing a través del planteamiento de un dilema sobre un caso de duelo acaecido en el aula.

Título actividad: «Dilema en el aula: la muerte y el duelo».

Etapa: Universitaria (adaptable a niveles inferiores)

Objetivos:

- Reflexionar sobre el papel del profesional de la educación ante la pedagogía de la muerte.

- Comprender la importancia del acompañamiento en los procesos de duelo al alumnado.

- Conocer recursos y estrategias para trabajar el tema de la muerte desde el contexto educativo.

Dilema: Lucas siempre ha sido uno de los líderes de la clase. Estudioso y a la par empático, conseguía el cariño y admiración de sus compañeros y profesores al mismo tiempo. Pero un día Lucas comenzó a cambiar. Se le veía triste y ausente unas veces; en otras ocasiones violento e impertinente; y por otras gastando bromas y riendo sin control. Algo le ocurría, pues no era el mismo de antaño. Tras una conversación con sus padres, descubrieron que el hermano pequeño de Lucas estaba gravemente enfermo y no sabrían si sobreviviría. Al poco tiempo, su hermano falleció y las actitudes de Lucas empeoraron ante la pérdida. Se da un caso de duelo en el aula con un alumno en plena adolescencia. En esta situación, interactúan los siguientes actores y cada uno de ellos con una perspectiva distinta sobre cómo proceder:

- Lucas: no acaba de asimilar lo sucedido y lo mismo llora o ríe, que actúa con violencia o desidia.

- Los padres de Lucas: les supera la pérdida de su hijo menor y tampoco saben cuál es la mejor forma para ayudar a Lucas al no ser capaces de reprimir el dolor.

- El tutor de Lucas: piensa que lo mejor es evitar el tema para no provocarle angustia a Lucas y sobreprotege al mismo perdonando sus actitudes.

- Los compañeros de clase: tienen curiosidad y quieren saber cómo se siente, por lo que preguntan directamente por la muerte de su hermano.

- El psicólogo escolar: quiere ayudar a Lucas a superar el proceso de duelo pero antes necesita saber cuál es la mejor forma de abordar el caso para evitar más complicaciones.

Instrucciones para su realización: Partiendo del dilema expuesto, la división de los alumnos se hará aleatoriamente. Cada grupo deberá reflexionar, analizar, debatir y proponer argumentos para defender su perspectiva, promoviendo así la empatía como una de las habilidades claves para la resolución positiva de los dilemas. Tras el tiempo consignado para realizar el análisis (en función del tiempo disponible de clase o de las sesiones que se le pueda dedicar a esta actividad), un miembro de cada grupo (ejerciendo de portavoz del mismo) representará mediante un role playing el papel asignando, dando argumentos para exponer sus circunstancias y deseos ante la situación planteada. Tras la puesta en común y 
de haber tomado notas de las aportaciones de cada grupo, se les pide que vuelvan a reunirse en sus grupos respectivos para comenzar a buscar vínculos y relaciones con el resto de personajes del dilema, con el fin de poder ir buscando soluciones, protocolos y estrategias que acerquen las perspectivas y posturas de los distintos grupos. Habrá una segunda ronda de intervenciones para comentar los posicionamientos y vínculos con otros grupos. La clave es subrayar esas sinergias e ir valorando las posibles actuaciones ante la situación de duelo en el aula. Se podrán hacer tantas vueltas al grupo de debate o al gran grupo como se considere necesario y oportuno. Una vez que se consideren las aportaciones concluidas, se indica al alumnado que vuelva a su grupo original. Se pide que cada grupo realice un análisis, interpretación y posible resolución del caso, con idea de compartir y discutir sobre las posibles soluciones. De esta manera, se ve como la situación de role playing planteada proporciona mecanismos, protocolos e instrumentos tanto para acompañar al alumnado en dichas situaciones como para que estos mejoren su control y gestión afectivo (relacionado con el sentir), cognitivo (relacionado con el saber) y conativo (relacionado con el actuar) ante la muerte.

Rol del educador: El docente deberá actuar como moderador, dando paso a los distintos agentes, controlando los tiempos de las intervenciones y sirviendo como hilo conductor entre las intervenciones de unos y otros portavoces. Es clave controlar los tiempos, la calidad y enriquecimiento del debate, y el interés y participación por parte del alumno. Además, deberá tener preparados recursos complementarios que sirvan como factores exploratorios (modificaciones, complicaciones o preguntas) para continuar con el debate y las argumentaciones siempre que quedan aspectos, temas, conceptos o problemáticas por abordar.

\section{Discusión y conclusiones}

Pese a que la muerte es un ámbito de estudio aceptado en diferentes disciplinas (Filosofía, Biología, Antropología, Sociología o Psicología), en la Pedagogía ha encontrado más barreras y obstáculos para su desarrollo, no teniendo un área propia para aportar sus conocimientos, instrumentos y posibilidades como ciencia. Se trata de un reto emergente que por su relevancia, se ha comenzado a demandar oficialmente (Herrán y Cortina, 2009), pidiendo la introducción de la Pedagogía de la Muerte en el currículo escolar. Sin embargo, ningún currículo oficial incluye expresamente a la muerte como ámbito formativo. Por esta razón, el cambio debe originarse como consecuencia natural de un proceso formativo necesario y profundo en este ámbito para la preparación de los futuros profesionales de la educación. Ya se han implementado programas para profesionales de la educación sobre la muerte que se han desarrollado internacionalmente (Harrawood, Doughty y Wilde, 2011), cuyos resultados apuntan a que dicho proceso formativo favoreció la comprensión sobre la finitud y la mortalidad del ser humano, a la par que redujo la ansiedad ante la muerte. Este aspecto es importante de considerar, ya que existe una relación significativa entre el grado de reflexión y conocimiento de los profesores sobre la muerte y su actitud favorable a la normalización educativa de la misma (Herrán y Cortina, 2006). Por esta razón, la muerte debe de desaparecer como un tabú e incluirse como ámbito formativo para el desarrollo, evolución y crecimiento de los educandos.

El fin es que la pedagogía de la muerte se generalice expresamente en el currículo, forme parte de los proyectos educativos de los centros y se desarrolle en las aulas de todos los niveles educativos como algo natural. Para ello el docente debe incluir a través de la (auto) formación, dentro de su proceso de reconstrucción identitaria profesional, a la muerte y a su pedagogía desde una percepción innovadora ligada a la vida. 


\section{Referencias}

Bandura, A. (1982). Teoría del aprendizaje social. Madrid: Espasa Calpe.

Bowie, L. (2000). Is there a place for death education in the primary curriculum? Pastoral Care, 1, 22-26.

Colomo, E. (2016). Pedagogía de la Muerte y Proceso de Duelo. Cuentos como Recurso Didáctico. REICE. Revista Iberoamericana sobre Calidad, Eficacia y Cambio en Educación, 14(2), 63-77. doi: 10.15366/reice2016.14.2.004

Colomo, E. y Oña, J.M. (2014). Pedagogía de la muerte. Las canciones como recurso didáctico. REICE. Revista Iberoamericana sobre Calidad, Eficacia y Cambio en Educación, 12(3), 109-121.

Contreras, P. (2010). Ser y saber en la formación didáctica del profesorado. Revista Interuniversitaria de Formación del Profesorado, 68 (24,2), 61-81.

Cortina, M. (2010). El cine como recurso didáctico de educación para la muerte: implicaciones formativas para el profesorado. Tesis Doctoral. Madrid: Universidad Autónoma de Madrid.

Daniels, H. y Bizar, M. (2005). Teaching the best practice way: Methods that matter. Portland: Stenhouse Publishers.

Engarhos, P., Talwar, V., Schleifer, M. y Renaud, S.J. (2013). Teachers' Attitudes and Experiences Regarding Death Education in the Classroom. Alberta Journal of Educational Research, 59 (1), 126-128.

Gaona, J.M. (2012a). El otro lado del túnel: Un camino hacia la luz en el umbral de la muerte. Madrid: La esfera de los libros.

Gaona, J.M. (2012b). ¿Son las experiencias cercanas a la muerte (ECM) la base empírica que demuestra la existencia del alma? Journal of Transpersonal Research, 4(2), 72-108.

Gaona, J.M. (2015). El límite: una profunda investigación sobre la consciencia, el cerebro y las experiencias cercanas a la muerte. Madrid: La esfera de los libros.Gillig, J.M. (1997). El cuento en pedagogía y en reeducación. México: Fondo de Cultura Económico.

Grup Xibeca (1995). Los dilemas morales. Un método para la educación en valores. Valencia: Nau Llibres.

Harrawood, L.K., Doughty, E.A. y Wilde, B. (2011). Death Education and Attitudes of Counselors-in-Training toward Death: An Exploratory Study. Counseling and Values, $56(1-2), 83-95$

Herrán, A. y Cortina, M. (2006). La muerte y su didáctica. Manual para educación infantil, primaria y secundaria. Madrid: Editorial Universitas.

Herrán, A. y Cortina, M. (2007). Introducción a una pedagogía de la muerte. Educación y Futuro, 17, 131-148.

Herrán, A. y Cortina, M. (2009). La muerte y su enseñanza. Diálogo Filosófico, 75, 499-516.

Lupión Cobos, T. y Gallego García, M.M. (2017). Compartiendo la mirada: una experiencia en práctica reflexiva para formación permanente. Revista Electrónica Interuniversitaria de Formación del Profesorado, 20(1), 127-144. doi: http://dx.doi.org/10.6018/reifop.20.1.244931 
Márquez-Cervantes, M.C. y Gaeta-González, M.L. (2017). Desarrollo de competencias emocionales en pre-adolescentes: el papel de padres y docentes. Revista Electrónica Interuniversitaria de Formación del Profesorado, 20(2), 221-235. doi: http://dx.doi.org/10.6018/reifop.20.1.232941

Niemiec, R.M.y Schulenberg, S.E. (2011). Understanding Death Attitudes: The Integration of Movies, Positive Psychology, and Meaning Management. Death Studies, 35 (5), 387407.

Pérez Gómez, Á.l. (2010). Aprender a educar. Nuevos desafíos para la formación docente. Revista Interuniversitaria de Formación del Profesorado, 68 (24, 2), 37-60.

Piñuel, J.L. y Gaitán, J.A. (1995). Metodología general: conocimiento científico e investigación en la comunicación social. Madrid: Síntesis.

Rodríguez, P. y Gorrayola, F. (2012). Propuestas didácticas para una pedagogía de la muerte desde la creatividad artística. REICE. Revista Iberoamericana sobre Calidad, Eficacia y Cambio en Educación, 10(2), 86-96.

Rodríguez, P., Herrán, A. y Cortina, M. (2015a). Educar y vivir teniendo en cuenta la muerte. Reflexiones y propuestas. Madrid: Pirámide.

Rodríguez, P., Herrán, A. y Cortina, M. (2015b). Pedagogía de la muerte mediante aprendizaje servicio. Educación XX1, 18(1), 189-212. doi: 10.5944/educXX1.18.1.12317

Sampedro, J.L. (2015). La vida perenne. Barcelona: Plaza \& Janés.

Santos Guerra, M.Á. (2010). La formación del profesorado en las instituciones que aprenden. Revista Interuniversitaria de Formación del Profesorado, 68 (24, 2), 175200.

Selfa, M., Fraga, F.J. y Berengué, I. (2015). Leer sobre la muerte en la biblioteca de aula: una experiencia práctica de lectura en un aula de Educación Infantil. Investigaciones Sobre Lectura, 3, 83-95.

Veneu, F. y Costa, M. (2016). Temas controvertidos en la clase: ¿estamos listos? Una pequeña investigación entre los profesores de ciencias. Revista Electrónica Interuniversitaria de Formación del Profesorado, 19(2), 89-100. doi: http://dx.doi.org/10.6018/reifop.19.2.253791 